\title{
EXISTENCE OF SOLUTION OF CONSTRAINED INTERVAL OPTIMIZATION PROBLEMS WITH REGULARITY CONCEPT
}

\author{
Priyanka Roy* And Geetanjali Panda
}

\begin{abstract}
Objective of this article is to study the conditions for the existence of efficient solution of interval optimization problem with inequality constraints. Here the active constraints are considered in inclusion form. The regularity condition for the existence of the Karush-Kuhn-Tucker point is derived. This condition depends on the interval-valued gradient function of active constraints. These are new concepts in the literature of interval optimization. gH-differentiability is used for the theoretical developments. gH-pseudo convexity for interval valued constrained optimization problems is introduced to study the sufficient conditions. Theoretical developments are verified through numerical examples.
\end{abstract}

Mathematics Subject Classification. 90C30, 49M05, 65G30.

Received December 14, 2019. Accepted June 7, 2020.

\section{INTRODUCTION}

The occurrence of vagueness in data of most of the real life optimization models is inevitable due to increasing in the complexity of nature and inherent subjective nature of human thought. In a general optimization problem, the impreciseness of various parameters lies either in the objective function or in the set of constraints. These parameters are accepted as intervals if the range of variation of these uncertain parameters are known in advance from the historical data. As a result, the objective and the constraints of the optimization model become interval valued functions and the model is called as interval optimization problem. A general constrained interval optimization model is stated as,

$$
\begin{array}{ll}
(\widehat{\mathrm{CP}}): \min & \hat{f}(x) \\
\text { Subject to } & x \in S \subseteq \mathbb{R}^{n}
\end{array}
$$

where $\hat{f}: \mathbb{R}^{n} \rightarrow I(\mathbb{R}), I(\mathbb{R})$ denotes the set of all closed intervals.

Conventional optimization techniques cannot be applied directly to solve $(\widehat{\mathrm{CP}})$ since the interval space is not linearly ordered. There are numerous studies in this direction over the years dealing with linear $[8-10,13,18,27]$ and nonlinear $[11,14,15,21]$ interval optimization models in both single and multi objective cases. Most of these methods follow a common process, which transforms $(\widehat{\mathrm{CP}})$ to a general optimization problem through different

Keywords. Interval valued function, interval optimization, generalized Hukuhara differentiability, Fritz-John conditions, KarushKuhn-Tucker conditions.

Department of Mathematics, Indian Institute of Technology Kharagpur, Kharagpur 721302, India.

*Corresponding author: proy180192@maths.iitkgp.ac.in 
scalarization techniques and determines the upper and lower bound of the objective function. Theoretical study for the existence of solution of a general nonlinear interval valued optimization problem is done by several researchers such as Wu [28,29], Bhurjee and Panda [5], Chalco-Cano et al. [7], Singh et al. [23, 24] and Osuna Gomez et al. $[19,20]$ etc. Bhurjee and Panda [5] studied the existence of the efficient solution of constrained interval optimization problem by parameterizing the interval function, which is also one type of scalarization process where the optimality conditions depend upon the structure of the transformed model. Wu [28, 29] derived the KKT optimality condition using $H$-derivative and LU convexity of interval valued function, which is dependent on endpoint functions. The $H$-derivative concept, introduced by Hukuhara [12], is used to study the optimality condition for interval valued objective function by many researchers. But this is very restrictive in nature because this concept is applicable only when two intervals are compared with respect to their lengths. Chalco-Cano et al. [7] studied KKT optimality condition using gH-derivative and LU convexity concept which are introduced by Stefanini and Bede [26] and Wu [28] respectively. Recently, gH-derivative is widely used for the theoretical developments of interval optimization problems (see Osuna Gomez et al. [19,20], Singh et al. $[23,24]$ etc.).

From the theoretical developments of interval optimization cited above, one may notice that the optimality conditions for $(\widehat{\mathrm{CP}})$ are expressed in the term of either the endpoint functions or their convex combination instead of focusing on the geometrical analysis of the descent structure as a whole and the conditions for active constraints are considered as equation type, which is either in parametric form or accepts the upper bound of the interval-valued constraints equal zero. These considerations are suitable in ideal situations but there is a chance of loss of data in complex situations since these processes take care only of the end point functions leaving intermediate points of the intervals untouched. Objective of this article is to address these shortfalls. Here, the conditions for the existence of solutions of $(\widehat{\mathrm{CP}})$ is studied concerning the descent property of the interval valued function which means, at the solution of $(\widehat{\mathrm{CP}})$, the set of feasible direction has empty intersection with the set of descent directions. Later, this concept is extended for $(\widehat{\mathrm{CP}})$ with inequality constraints. Sufficient conditions are derived under pseudo-convex property of interval valued function. Conditions for active constraints are taken as inclusion type and regularity conditions are considered using the linear independence property of interval vectors. These are the new contributions to the theory of interval optimization.

This article is organized as follows: Section 2 presents the pre-requisites on interval analysis. In Section 3, the concept of feasible descent direction at a point and the conditions for the existence of solution of $(\widehat{\mathrm{CP}})$ are studied. Sufficient conditions are derived using gH-pseudo convex property of interval valued function. Concept of this section is extended to $(\widehat{\mathrm{CP}})$ with interval valued inequality constraints in Section 4 . Regularity condition is studied with the help of linear independent interval vectors, which helps to derive the sufficient condition.

\section{Prerequisites}

Let $I(\mathbb{R})$ be the set of all closed intervals on the real line $\mathbb{R}$ and $\hat{a} \in I(\mathbb{R})$ be the closed interval of the form $[\underline{a}, \bar{a}]$ with $\underline{a} \leq \bar{a}$, where $\underline{a}, \bar{a}$ denote the lower and upper bound of $\hat{a}$ respectively. Any real number $x$ can be expressed as a degenerate interval denoted by $\hat{x}$ as $\hat{x}=[x, x]$ or $x \cdot \hat{I}$, where $\hat{I}=[1,1] . \hat{0}=[0,0]$ denotes the null interval. Interior of $\hat{a} \in I(\mathbb{R}) \operatorname{denoted} \operatorname{by} \operatorname{int}(\hat{a})$ and $\operatorname{int}(\hat{a}) \triangleq(\underline{a}, \bar{a})$. Denote an index set $\Lambda_{n}=\{1,2, \ldots, n\}$.

Addition of two intervals $\hat{a}=[\underline{a}, \bar{a}], \hat{b}=[\underline{b}, \bar{b}] \in I(\mathbb{R})$ is, $\hat{a} \oplus \hat{b}=[\underline{a}+\underline{b}, \bar{a}+\bar{b}]$.

For $\alpha \in \mathbb{R}$,

$$
\alpha \hat{a}= \begin{cases}{[\alpha \underline{a}, \alpha \bar{a}]} & \text { if } \alpha \geq 0, \\ {[\alpha \bar{a}, \alpha \underline{a}]} & \text { if } \alpha \leq 0 .\end{cases}
$$

According to this rule, $\hat{a} \ominus \hat{b}=\hat{a} \oplus(-1) \hat{b}=[\underline{a}-\bar{b}, \bar{a}-\underline{b}]$, which says that $\hat{a} \ominus \hat{a}$ is not necessarily $\hat{0}$. Hence additive inverse of an interval may not exist in the interval space. To overcome this difficulty, Stefanini [25] introduced 
the concept of generalized Hukuhara difference (gH difference) between two intervals $\hat{a}$ and $\hat{b}$, as

$$
\hat{a} \ominus_{\mathrm{gH}} \hat{b}=\hat{c} \Leftrightarrow\left\{\begin{array}{l}
\hat{a}=\hat{b} \oplus \hat{c} \\
\text { or } \\
\hat{a} \oplus(-1) \hat{c}=\hat{b}
\end{array}\right.
$$

which is equivalent to $\hat{a} \ominus_{\mathrm{gH}} \hat{b}=[\min \{\underline{a}-\underline{b}, \bar{a}-\bar{b}\}, \max \{\underline{a}-\underline{b}, \bar{a}-\bar{b}\}]$.

This is the most generalized concept of interval difference used in interval calculus. The property of this gH-difference operation are as follows:

(i) $\ominus_{\mathrm{gH}} \hat{a}=\hat{0} \ominus_{\mathrm{gH}} \hat{a}=[\min \{0-\underline{a}, 0-\bar{a}\}, \max \{0-\underline{a}, 0-\bar{a}\}]=[-\bar{a},-\underline{a}]=(-1) \odot \hat{a}$,

(ii) $\hat{a} \ominus_{\mathrm{gH}} \hat{a}=\hat{0}$.

$I(\mathbb{R})$ is not a totally ordered set. Several partial orderings exist in the literature of interval analysis (see [17]). Following interval ordering from [5] is often used for solving interval optimization problem.

Definition 2.1. For $\hat{a}=[\underline{a}, \bar{a}], \hat{b}=[\underline{b}, \bar{b}] \in I(\mathbb{R}), \hat{a} \preceq \hat{b} \Leftrightarrow \underline{a} \leq \underline{b}$ and $\bar{a} \leq \bar{b} ; \hat{a} \preceq \hat{b} \Leftrightarrow \hat{a} \preceq \hat{b}$ and $\hat{a} \neq \hat{b}$; $\hat{a} \prec \hat{b} \Leftrightarrow \underline{a}<\underline{b}$ and $\bar{a}<\bar{b}$. The interval order relations " $\succeq$ ", " $\succeq$ " and " $\succ$ " are defined in a similar way by reverting the inequalities.

The product $d^{T} \hat{a}$ where $d \in \mathbb{R}^{n}$ and $\hat{a}=\left(\hat{a}_{1}, \ldots, \hat{a}_{n}\right)^{T}, \hat{a}_{i} \in I(\mathbb{R})$ is,

$$
d^{T} \hat{a}=d_{1} \hat{a}_{1} \oplus d_{2} \hat{a}_{2} \oplus \cdots \oplus d_{n} \hat{a}_{n} \triangleq \sum_{i=1}^{n} d_{i} \hat{a}_{i}
$$

One may note that $\hat{a}^{T} d=d^{T} \hat{a}$. In $I(\mathbb{R})$, the norm $(\|\cdot\|)$ of an interval $\hat{a}$ is defined as $\|\hat{a}\|=\max \{|\underline{a}|,|\bar{a}|\}$, which is associated with the metric structure $D(\hat{a}, 0)=\|\hat{a}\|$ and $D(\hat{a}, \hat{b})=\max \{|\underline{a}-\underline{b}|,|\bar{a}-\bar{b}|\}$, which is the Hausdorff distance between intervals (see [26]).

Limit and continuity of an interval valued function is understood in the sense of metric structure of $\mathrm{gH}$ difference using Hausdorff distance between intervals (see [26]). Some existing results of gH-differentiable interval valued function, $\hat{f}(x)=[\underline{f}(x), \bar{f}(x)]$, where $\underline{f}, \bar{f}: \mathbb{R}^{n} \rightarrow \mathbb{R}, \underline{f}(x) \leq \bar{f}(x) \forall x$, are provided in this section.

Definition 2.2 ([26]). Generalized Hukuhara derivative of $\hat{f}:\left(t_{1}, t_{2}\right) \subseteq \mathbb{R} \rightarrow I(\mathbb{R})$ at $x \in\left(t_{1}, t_{2}\right)$ is defined as $\hat{f}^{\prime}(x)=\lim _{h \rightarrow 0} \frac{\hat{f}(x+h) \ominus_{\mathrm{gH}} \hat{f}(x)}{h}$.

Chalco et al. [6] justify that the concept of gH difference is same as Markov difference $\left(\ominus_{M}\right)$, which was introduced by Markov [16] in the set of intervals. Following results from [16] are used for some of the theoretical developments in this article.

Theorem $2.3([16])$. If $\hat{f}: \mathbb{R} \rightarrow I(\mathbb{R})$ is continuous in $\Delta=[\alpha, \beta]$ and differentiable in $(\alpha, \beta)$, then $\hat{f}(\beta) \ominus_{M}$ $\hat{f}(\alpha) \subset \hat{f}^{\prime}(\Delta)(\beta-\alpha)$, where $\hat{f}^{\prime}(\Delta)=\cup_{\xi \in \Delta} \hat{f}^{\prime}(\xi)$.

Definition 2.4 (Generalized Hukuhara Differentiability in Higher Dimension, [19]). Let $\hat{f}: X \subseteq \mathbb{R}^{n} \rightarrow I(\mathbb{R})$ and $x=\left(x_{1}, x_{2}, \ldots, x_{n}\right)^{T}$ be a fixed element of $X$. Consider the interval valued function $\hat{\psi}_{i}\left(x_{i}\right) \triangleq \hat{f}\left(x_{1}, x_{2}, \ldots x_{i-1}, x_{i}, x_{i+1}, \ldots, x_{n}\right)$.

If $\lim _{h_{i} \rightarrow 0} \frac{\hat{\psi}_{i}\left(x_{i}+h_{i}\right) \ominus_{\mathrm{gH}} \hat{\psi}_{i}\left(x_{i}\right)}{h_{i}}$ exists, then we say that the partial derivative of $\hat{f}$ with respect to $x_{i}$ exists at $x$ and the limiting value is denoted by $\frac{\partial \hat{f}(x)}{\partial x_{i}}$. 
Relation between the existence of gH derivative of single variable interval valued function $\hat{f}:\left(t_{1}, t_{2}\right) \rightarrow I(\mathbb{R})$ and the existence of derivative of its endpoint function $f$ and $\bar{f}$ is studied in Theorem 1 of [19]. In the light of this theorem the existence of gH partial derivative of $\hat{f}: X \subseteq \mathbb{R}^{n} \rightarrow I(\mathbb{R})$ and the existence of the partial derivatives of its endpoint functions can be interpreted as follows.

Existence of the partial derivative of $\hat{f}: X \subseteq \mathbb{R}^{n} \rightarrow I(\mathbb{R})$ with respect to $x_{i}$ at $x$ is equivalent to

(a) Partial derivatives of $\underline{f}$ and $\bar{f}$ with respect to $x_{i}$ at $x$ exist and

$$
\frac{\partial \hat{f}(x)}{\partial x_{i}}=\left[\min \left\{\frac{\partial \underline{f}(x)}{\partial x_{i}}, \frac{\partial \bar{f}(x)}{\partial x_{i}}\right\}, \quad \max \left\{\frac{\partial \underline{f}(x)}{\partial x_{i}}, \frac{\partial \bar{f}(x)}{\partial x_{i}}\right\}\right] .
$$

(b) The lateral partial derivatives of $\underline{f}$ and $\bar{f}$ at $x$ with respect to $x_{i}$. That is, $\left(\frac{\partial f(x)}{\partial x_{i}}\right)_{-},\left(\frac{\partial f(x)}{\partial x_{i}}\right)_{+}$and $\left(\frac{\partial \bar{f}(x)}{\partial x_{i}}\right)_{-}$, $\left(\frac{\partial \bar{f}(x)}{\partial x_{i}}\right)_{+}$exist, and satisfy $\left(\frac{\partial \underline{f}(x)}{\partial x_{i}}\right)_{-}=\left(\frac{\partial \bar{f}(x)}{\partial x_{i}}\right)_{+} ;\left(\frac{\partial \underline{f}(x)}{\partial x_{i}}\right)_{+}=\left(\frac{\partial \bar{f}(x)}{\partial x_{i}}\right)_{-}$. Moreover,

$$
\begin{aligned}
& \frac{\partial \hat{f}(x)}{\partial x_{i}}=\left[\min \left\{\left(\frac{\partial \underline{f}(x)}{\partial x_{i}}\right)_{-},\left(\frac{\partial \bar{f}(x)}{\partial x_{i}}\right)_{-}\right\}, \quad \max \left\{\left(\frac{\partial \underline{f}(x)}{\partial x_{i}}\right)_{-},\left(\frac{\partial \bar{f}(x)}{\partial x_{i}}\right)_{-}\right\}\right] \\
& =\left[\min \left\{\left(\frac{\partial \underline{f}(x)}{\partial x_{i}}\right)_{+},\left(\frac{\partial \bar{f}(x)}{\partial x_{i}}\right)_{+}\right\}, \quad \max \left\{\left(\frac{\partial \underline{f}(x)}{\partial x_{i}}\right)_{+},\left(\frac{\partial \bar{f}(x)}{\partial x_{i}}\right)_{+}\right\}\right] \text {. }
\end{aligned}
$$

Gradient of interval valued function at a point $x \in \mathbb{R}^{n}$ is an interval vector, which is denoted by

$$
\nabla \hat{f}(x) \triangleq\left(\frac{\partial \hat{f}(x)}{\partial x_{1}}, \frac{\partial \hat{f}(x)}{\partial x_{2}}, \ldots, \frac{\partial \hat{f}(x)}{\partial x_{n}}\right)^{T}
$$

Definition $2.5([22]) . \hat{f}: X \rightarrow I(\mathbb{R}), X$ is an open subset of $\mathbb{R}^{n}$, is said to be a gH differentiable at $x_{0} \in X$ if $\nabla \hat{f}\left(x_{0}\right)$ exists and $\lim _{\|h\| \rightarrow 0} \frac{\hat{w}\left(\hat{f}\left(x_{0}\right) ; h\right) \ominus_{\mathrm{gH}} h^{T} \nabla \hat{f}\left(x_{0}\right)}{\|h\|}=\hat{0}$, where $\hat{w}\left(\hat{f}\left(x_{0}\right) ; h\right) \triangleq \hat{f}\left(x_{0}+h\right) \ominus_{\mathrm{gH}} \hat{f}\left(x_{0}\right)$. This can be restated using error function. $\hat{f}$ is said to be gH differentiable at $x_{0} \in X$ if $\nabla \hat{f}\left(x_{0}\right)$ exists and there exists an interval valued error function $\hat{E}_{x_{0}}: \mathbb{R}^{n} \rightarrow I(\mathbb{R})$ satisfying $\lim _{\|h\| \rightarrow 0} \hat{E}_{x_{0}}(h)=\hat{0}$ where $\hat{E}_{x_{0}}(h)=\frac{\hat{w}\left(\hat{f}\left(x_{0}\right) ; h\right) \ominus_{\mathrm{gH}} h^{T} \nabla \hat{f}\left(x_{0}\right)}{\|h\|}$. In other words, $\exists \delta>0$ very small such that $\hat{w}\left(\hat{f}\left(x_{0}\right) ; h\right) \ominus_{\mathrm{gH}} h^{T} \nabla \hat{f}(x)=\|h\| \hat{E}_{x_{0}}(h)$ holds for $0<\|h\|<\delta$. Using the concept of $\mathrm{gH}$ difference (2.1), this expression can be expressed in an equivalent form as follows:

$$
\begin{array}{ll}
\text { either } & \hat{w}\left(\hat{f}\left(x_{0}\right) ; h\right)=h^{T} \nabla \hat{f}\left(x_{0}\right) \oplus\left(\|h\| \hat{E}_{x_{0}}(h)\right) \\
\text { or } & \hat{w}\left(\hat{f}\left(x_{0}\right) ; h\right) \oplus(-1)\left(\|h\| \hat{E}_{x_{0}}(h)\right)=h^{T} \nabla \hat{f}\left(x_{0}\right)
\end{array}
$$

where $h^{T} \nabla \hat{f}\left(x_{0}\right)=\sum_{i=1}^{n} h_{i} \frac{\partial \hat{f}\left(x_{0}\right)}{\partial x_{i}}$.

Theorem $2.6([22])$. Suppose $\hat{f}: \mathbb{R}^{n} \rightarrow I(\mathbb{R})$ is an interval valued $\mathrm{gH}$-differentiable function at $x_{0}$ and $u$ : $R \rightarrow \mathbb{R}^{n}$ be differentiable at " $a$ " with total derivative $\left(u_{1}^{\prime}(a), u_{2}^{\prime}(a), \ldots, u_{n}^{\prime}(a)\right)^{T}$. If $x_{0}=u(a)$ then the composite function $\hat{\phi} \triangleq \hat{f} \circ u: \mathbb{R} \rightarrow I(\mathbb{R})$ is $\mathrm{gH}$ differentiable at $a$, and $\hat{\phi}^{\prime}(a)=\sum_{i=1}^{n} u_{i}^{\prime}(a) \frac{\partial \hat{f}\left(x_{0}\right)}{\partial x_{i}}$.

In the rest part of the article, the following notations are used to simplify the expressions. 


\section{Notations}

$$
\begin{aligned}
& \nabla \hat{f}(x) \triangleq\left(\frac{\partial \hat{f}(x)}{\partial x_{1}}, \frac{\partial \hat{f}(x)}{\partial x_{2}}, \ldots, \frac{\partial \hat{f}(x)}{\partial x_{n}}\right)^{T} \\
& \frac{\partial \hat{f}(x)}{\partial x_{i}} \triangleq\left[\left(\frac{\partial \hat{f}(x)}{\partial x_{i}}\right)_{\min },\left(\frac{\partial \hat{f}(x)}{\partial x_{i}}\right)_{\max }\right]
\end{aligned}
$$

$\Lambda_{n}=\{1,2, \ldots, n\}$ denotes the index set.

From (2.2) and (2.3),

$$
\begin{aligned}
& \left(\frac{\partial \hat{f}(x)}{\partial x_{i}}\right)_{\min } \triangleq \min \left\{\frac{\partial f(x)}{\partial x_{i}}, \frac{\partial \bar{f}(x)}{\partial x_{i}}\right\} \text { or } \min \left\{\left(\frac{\partial f(x)}{\partial x_{i}}\right)_{-},\left(\frac{\partial \bar{f}(x)}{\partial x_{i}}\right)_{-}\right\} \text {or } \min \left\{\left(\frac{\partial f(x)}{\partial x_{i}}\right)_{+},\left(\frac{\partial \bar{f}(x)}{\partial x_{i}}\right)_{+}\right\}, \quad i \in \Lambda_{n} . \\
& \left(\frac{\partial \hat{f}(x)}{\partial x_{i}}\right)_{\max } \triangleq \max \left\{\frac{\partial \bar{f}(x)}{\partial x_{i}}, \frac{\partial \bar{f}(x)}{\partial x_{i}}\right\} \text { or } \max \left\{\left(\frac{\partial \bar{f}(x)}{\partial x_{i}}\right)_{-},\left(\frac{\partial \bar{f}(x)}{\partial x_{i}}\right)_{-}\right\} \text {or } \max \left\{\left(\frac{\partial f(x)}{\partial x_{i}}\right)_{+},\left(\frac{\partial \bar{f}(x)}{\partial x_{i}}\right)_{+}\right\}, \quad i \in \Lambda_{n} .
\end{aligned}
$$

\section{Existence OF SOlution of $(\widehat{\mathrm{CP}})$}

Consider the problem $(\widehat{\mathrm{CP}})$, where $S$ is an open, convex subset of $R^{n}$. Assume that $\hat{f}: \mathbb{R}^{n} \rightarrow I(\mathbb{R})$ is gH differentiable and all the partial derivatives of $\hat{f}$ are continuous on $S$ throughout the rest part of the article.

Definition 3.1 ([19]). A point $x^{*} \in S$ is called a (local) strong efficient solution of $(\widehat{\mathrm{CP}})$ if $\nexists x \in S$ (i.e. $\exists \delta>0$, $x \in B\left(x^{*}, \delta\right) \cap S$ ) such that $\hat{f}(x) \preceq \hat{f}\left(x^{*}\right)$, where $B\left(x^{*}, \delta\right)$ denotes the neighbourhood of $x^{*}$ with radius $\delta$.

A point $x^{*} \in S$ is called a (local) efficient solution of ( $\left.\widehat{\mathrm{CP}}\right)$ if $\nexists x \in S$ (i.e. $\left.\exists \delta>0, x \in B\left(x^{*}, \delta\right) \cap S\right)$ such that $\hat{f}(x) \preceq \hat{f}\left(x^{*}\right)$.

A point $x^{*} \in S$ is called (local) weak efficient solution of ( $\left.\widehat{\mathrm{CP}}\right)$ if $\nexists x \in S$ (i.e. $\left.\exists \delta>0, x \in B\left(x^{*}, \delta\right) \cap S\right)$ such that $\hat{f}(x) \prec \hat{f}\left(x^{*}\right)$.

For a given point $x \in S$, if $\hat{f}(x+\alpha d)(\prec$ or $\preceq$ or $\preceq) \hat{f}(x)$ holds for some non zero $d \in \mathbb{R}^{n}$, then $x+\alpha d$ is the point of improvement of the objective function $\hat{f}(x)$ of $(\widehat{\mathrm{CP}})$ and $d$ is a descent direction at $x$. In this article we accept " $\prec$ " to define the descent direction at a point.

Definition 3.2. A non zero vector $d \in \mathbb{R}^{n}$ is said to be a descent direction of $\hat{f}$ at $x \in S$ with respect to " $\prec$ " if there exists some $\delta>0$ such that $\hat{f}(x+\alpha d) \prec \hat{f}(x) \forall \alpha \in(0, \delta)$.

For a given point $x \in S$, denote the set of descent direction by $\mathscr{D}_{0}$ at a point $x$ with respect to “ $\prec$.

$$
\mathscr{D}_{0}(x) \triangleq\left\{d \in \mathbb{R}^{n}: \hat{f}(x+\alpha d) \prec \hat{f}(x) \quad \forall \alpha \in(0, \delta)\right\} .
$$

Following result is a consequence of Theorems 2.3 and 2.6 which will be used further to derive descent direction.

Lemma 3.3. For any $x, y \in S$,

$$
\hat{f}(y) \ominus_{\mathrm{gH}} \hat{f}(x) \subset \underset{c \in \mathrm{L} . \mathrm{S}\{x, y\}}{\cup}(y-x)^{T} \nabla \hat{f}(c)
$$

where L.S $\{x, y\}$ denotes the line segment joining $x$ and $y$. 
Proof. For $x, y \in S$, denote $\gamma(t)=x+t(y-x), t \in[0,1] . \gamma(t) \in S$, since $S$ is a convex set.

Let $\hat{\phi}:[0,1] \rightarrow I(\mathbb{R})$ be defined by $\hat{\phi}(t)=\hat{f}\left(\gamma_{1}(t), \gamma_{2}(t), \ldots \gamma_{n}(t)\right)$, where

$\gamma_{i}(t)=x_{i}+t\left(y_{i}-x_{i}\right), \forall i=1,2, \ldots, n, t \in[0,1] . \hat{\phi}$ is gH-differentiable, which follows from Theorem 2.6.

Therefore $\hat{\phi}^{\prime}(t)=\sum_{i=1}^{n} \gamma_{i}^{\prime}(t) \frac{\partial \hat{f}(\gamma(t))}{\partial \gamma_{i}}=\sum_{i=1}^{n}\left(y_{i}-x_{i}\right) \frac{\partial \hat{f}(\gamma(t))}{\partial \gamma_{i}}=(y-x)^{T} \nabla \hat{f}(\gamma(t))$.

From Theorem 2.3, $\hat{\phi}(1) \ominus_{\mathrm{gH}} \hat{\phi}(0) \subset \underset{\theta \in[0,1]}{\cup} \hat{\phi}^{\prime}(\theta)$. Here $\hat{\phi}(1)=\hat{f}(y)$ and $\hat{\phi}(0)=\hat{f}(x)$. Hence (3.1) follows, where $c=x+\theta(y-x)$ for some $\theta$ and $\hat{\phi}^{\prime}(\theta)=(y-x)^{T} \nabla \hat{f}(c)$.

Theorem 3.4. If $d^{T} \nabla \hat{f}(x) \prec \hat{0}$ for some non zero $d \in \mathbb{R}^{n}$, then $d$ is a descent direction of $\hat{f}$ at $x$.

Proof. $d^{T} \nabla \hat{f}(x): \mathbb{R}^{n} \rightarrow I(\mathbb{R})$ is continuous at $x$ since $\frac{\partial \hat{f}(x)}{\partial x_{i}}$ is continuous for every $i \in \Lambda_{n}$. Since $d^{T} \nabla \hat{f}(x) \prec \hat{0}$, so $0 \notin \operatorname{int}\left(d^{T} \nabla \hat{f}(x)\right)$. Hence

$$
d^{T} \nabla \hat{f}(x+\alpha d) \prec \hat{0} \quad \forall \alpha \in(0, \delta), \quad \text { some } \quad \delta>0 .
$$

In the inclusion result (3.1) of Lemma 3.3, replacing $y$ by $x+\alpha d$, we have

$$
\hat{f}(x+\alpha d) \ominus_{\mathrm{gH}} \hat{f}(x) \subset \cup_{c \in} \quad \text { L.S }\{x, x+\alpha d\} \alpha d^{T} \nabla \hat{f}(c) .
$$

From (3.2) and (3.3), $\hat{f}(x+\alpha d) \ominus_{\mathrm{gH}} \hat{f}(x) \prec \hat{0}$. Hence $\hat{f}(x+\alpha d) \prec \hat{f}(x)$ follows. Therefore $d$ is the descent direction at $x$.

Let $\mathscr{D}_{1}$ denotes the set of descent directions at a point $x \in S$ which follows Theorem 3.4.

$$
\mathscr{D}_{1}(x) \triangleq\left\{d \in \mathbb{R}^{n}: d^{T} \nabla \hat{f}(x) \prec \hat{0}\right\} .
$$

Lemma 3.5. At $x \in S, \mathscr{D}_{1}(x) \subseteq \mathscr{D}_{0}(x)$.

Proof. Let $d \in \mathscr{D}_{1}(x)$. Then $d^{T} \nabla \hat{f}(x) \prec \hat{0}$. Since all the partial derivatives of $\hat{f}$ are continuous, $d^{T} \nabla \hat{f}(x)$ is continuous. Therefore $\exists \delta^{\prime}>0$ such that $d^{T} \nabla \hat{f}(x+\alpha d) \prec \hat{0} \forall \alpha \in\left(0, \delta^{\prime}\right)$. Therefore from (3.3), $\hat{f}(x+\alpha d) \prec \hat{f}(x)$ holds $\forall \alpha \in\left(0, \delta^{\prime}\right)$. Therefore $d \in \mathscr{D}_{0}(x)$. Hence

$$
\mathscr{D}_{1}(x) \subseteq \mathscr{D}_{0}(x)
$$

Hence from (3.4), the result follows.

Definition 3.6. A non zero vector $d \in \mathbb{R}^{n}$ is said to be a feasible direction at $x \in S$ if there exists some $\delta_{1}>0$ such that $x+\alpha d \in S \forall \alpha \in\left(0, \delta_{1}\right)$.

Let $\mathscr{F}(x)$ denote the set of feasible directions at $x \in S$. i.e.

$$
\mathscr{F}(x) \triangleq\left\{d \in \mathbb{R}^{n}: x+\alpha d \in S \quad \forall \alpha \in\left(0, \delta_{1}\right)\right\} .
$$

Some necessary conditions for the existence of weak efficient solution for $(\widehat{\mathrm{CP}})$ are studied below.

Theorem 3.7. If $x^{*}$ is local weak efficient solution of $(\widehat{\mathrm{CP}})$, then $\mathscr{F}\left(x^{*}\right) \bigcap \mathscr{D}_{0}\left(x^{*}\right)=\phi$.

Proof. Let $x^{*} \in S$ be local weak efficient solution of $(\widehat{\mathrm{CP}})$. If possible let $\mathscr{F}\left(x^{*}\right) \bigcap \mathscr{D}_{0}\left(x^{*}\right) \neq \phi$. There exists a non zero direction $d \in \mathscr{F}\left(x^{*}\right) \bigcap \mathscr{D}_{0}\left(x^{*}\right)$. Therefore from Definition 3.6, $\exists \delta_{1}>0$ such that $x^{*}+\alpha d \in S \forall \alpha \in\left(0, \delta_{1}\right)$ and from Definition 3.2, $\exists \delta_{2}>0$ such that $\hat{f}\left(x^{*}+\alpha d\right) \prec \hat{f}\left(x^{*}\right) \forall \alpha \in\left(0, \delta_{2}\right)$. Choose $\delta:=\min \left\{\delta_{1}, \delta_{2}\right\}$ Hence $\exists x \in B\left(x^{*}, \alpha\right) \bigcap S$, such that $\hat{f}(x) \prec \hat{f}\left(x^{*}\right) \forall \alpha \in(0, \delta)$. This contradicts the assumption that $x^{*} \in S$ is a local weak efficient solution of $(\widehat{\mathrm{CP}})$. Hence $\mathscr{F}\left(x^{*}\right) \bigcap \mathscr{D}_{0}\left(x^{*}\right)=\phi$. 
Corollary 3.8. If $x^{*}$ is local weak efficient solution of $(\widehat{\mathrm{CP}})$, then $\mathscr{F}\left(x^{*}\right) \bigcap \mathscr{D}_{1}\left(x^{*}\right)=\phi$.

Proof. All the conditions of Lemma 3.5 are satisfied. Therefore at $x^{*} \in S$, from Theorem 3.5, $\mathscr{D}_{1}\left(x^{*}\right) \subseteq \mathscr{D}_{0}\left(x^{*}\right)$ hold. Again from Theorem 3.7, it follows that $\mathscr{F}\left(x^{*}\right) \bigcap \mathscr{D}_{0}\left(x^{*}\right)=\phi$. Hence the required result follows.

Generalized convexity plays an important role to ensure the optimal solution of classical optimization problem. Sufficient conditions for the existence of solution for interval optimization problem are studied by several researchers using convexity $[2,28,29]$ or pseudo convexity assumption $[1,30]$ on either endpoint functions or real valued parametric form of interval valued function. In this article we have defined pseudo convexity for interval valued function (not necessarily dependent on endpoint function) using generalized Hukuhara differentiability. This concept is used to justify the existence of weak efficient solution. We accept the interval ordering " $\prec$ " to define pseudo convexity of interval valued function at a point. However in a similar way other interval ordering can be used to define pseudo convexity.

Definition 3.9. A gH-differentiable interval valued function $\hat{f}: \mathbb{R}^{n} \rightarrow I(\mathbb{R})$ is said to be gH-pseudo convex at $\bar{x} \in S$ if $\hat{f}(x) \prec \hat{f}(\bar{x})$, then $(x-\bar{x})^{T} \nabla \hat{f}(\bar{x}) \prec \hat{0}$ holds for each $x \in S$.

Note 3.10. The pseudo convexity of endpoint functions does not depend on the gH-pseudo convexity of interval valued function. This is justified in the following example.

Example 3.11. Consider $\hat{f}\left(x_{1}, x_{2}\right)=[1,4] x_{1}^{2} \oplus[1,3] x_{1} \oplus[2,4] x_{2}$. At $x=(0,0)^{T}, \hat{f}$ is gH differentiable and $\nabla \hat{f}(0,0)=([1,3],[2,4])^{T}$ though $\underline{f}$ and $\bar{f}$ are not differentiable at $x=(0,0)^{T}$.

Consider an another point $y$ such that $\hat{f}(y) \prec \hat{f}(x)$ hold. Then

$$
[1,4] y_{1}^{2} \oplus[1,3] y_{1} \oplus[2,4] y_{2} \prec \hat{0} .
$$

Using (3.5), $(y-x)^{T} \nabla \hat{f}(x)=y_{1}[1,3] \oplus y_{2}[2,4] \prec \ominus_{\mathrm{gH}}[1,4] y_{1}^{2} \prec \hat{0}$.

Therefore $\hat{f}(y) \prec \hat{f}(x) \Rightarrow(y-x)^{T} \nabla \hat{f}(x) \prec \hat{0}$ hold. Hence $\hat{f}$ is gH-pseudo convex at $(0,0)^{T}$.

Theorem 3.12. Suppose $\hat{f}: \mathbb{R}^{n} \rightarrow I(\mathbb{R})$ is gH-pseudo-convex at $x^{*} \in S$ and $\mathscr{F}\left(x^{*}\right) \bigcap \mathscr{D}_{1}\left(x^{*}\right)=\phi$. Then $x^{*}$ is a local weak efficient solution of $(\widehat{\mathrm{CP}})$.

Proof. Suppose $x^{*}$ is not a local weak efficient solution of $(\widehat{\mathrm{CP}})$. Then there exists $\bar{x} \in S$ such that $\hat{f}(\bar{x}) \prec \hat{f}\left(x^{*}\right)$. Since $\hat{f}$ is pesudo convex at $x^{*}$,

$$
\left(\bar{x}-x^{*}\right)^{T} \nabla \hat{f}\left(x^{*}\right) \prec \hat{0} \Rightarrow\left(\bar{x}-x^{*}\right) \in \mathscr{D}_{1}\left(x^{*}\right) .
$$

Let $d=\bar{x}-x^{*}$. Then $x=x^{*}+\lambda\left(\bar{x}-x^{*}\right) \in S$ for $\lambda \in(0, \delta)$ since $S$ is an open convex set. Therefore $\left(\bar{x}-x^{*}\right) \in \mathscr{F}\left(x^{*}\right)$ and $\mathscr{F}\left(x^{*}\right) \bigcap \mathscr{D}_{1}\left(x^{*}\right) \neq \phi$, which contradicts the hypothesis.

Hence $x^{*}$ is a local weak efficient solution of $(\widehat{\mathrm{CP}})$.

\section{Existence of SOlution OF $(\widehat{\mathrm{CP}})$ With INEQUALity CONSTRAints}

Consider the following interval valued minimization problem with inequality constraints.

$$
\begin{aligned}
(\widehat{\mathrm{CP}})_{1}: \min & \hat{f}(x) \\
\text { Subject to: } & \hat{g}_{j}(x) \precsim \hat{0} ; \quad j \in \Lambda_{m}, \quad \text { where } \quad \Lambda_{m}=\{1,2, \ldots, m\} \\
& x \in X \subseteq \mathbb{R}^{n},
\end{aligned}
$$

where $\hat{f}, \hat{g}_{j}: \mathbb{R}^{n} \rightarrow I(\mathbb{R}), \forall j \in \Lambda_{m}, X$ is an open subset of $\mathbb{R}^{n}$. Here the feasible set will be specified as $S:=\left\{x \in X: \hat{g}_{j}(x) \preceq \hat{0} ; j \in \Lambda_{m}\right\}$. Given a point $\bar{x} \in S$, denote $\Lambda^{\text {ac }}(\bar{x}):=\left\{j: 0 \in \hat{g}_{j}(\bar{x}), j \in \Lambda_{m}\right\}$ 
and $\left.\Lambda^{\text {in }}(\bar{x}):=\left\{j: \hat{g}_{j}(\bar{x}) \prec \hat{0}, j \in \Lambda_{m}\right)\right\} . \hat{g}_{j}(x) \preceq \hat{0}$ and $0 \in \hat{g}_{j}(\bar{x}), j \in \Lambda^{\text {ac }}(\bar{x}) \Rightarrow$ either $\bar{g}_{j}(\bar{x})=0$ or $\underline{g}_{j}(\bar{x})=0 ; \bar{g}_{j}(\bar{x})=0$.

The following assumptions are considered for $(\widehat{\mathrm{CP}})_{1}$ to study the theoretical results. $\hat{f}$ and $\hat{g}_{j}: \mathbb{R}^{n} \rightarrow I(\mathbb{R})$, $j \in \Lambda^{\text {ac }}(x)$ are gH differentiable over $X$ and all the partial derivatives of $\hat{g}_{j}, j \in \Lambda^{\text {ac }}(x)$ are continuous. $\hat{g}_{j}, j \in$ $\Lambda^{\text {in }}(x)$ are continuous.

Denote $\overline{\mathscr{F}}(x) \triangleq\left\{d \in \mathbb{R}^{n}: d^{T} \nabla \hat{g}_{j}(x) \prec \hat{0}, j \in \Lambda^{\text {ac }}(x)\right\}$ at any point $x \in S$.

\subsection{Necessary conditions for weak efficient point}

In this section some necessary conditions for the existence of weak efficient point of $(\widehat{\mathrm{CP}})_{1}$ are studied.

Lemma 4.1. At $x \in S, \overline{\mathscr{F}}(x) \subseteq \mathscr{F}(x)$.

Proof. Let $d \in \overline{\mathscr{F}}(x)$. Then $d^{T} \nabla \hat{g}_{j}(x) \prec \hat{0}, \forall j \in \Lambda^{\mathrm{ac}}(x)$. Using Theorem 3.4, $d$ is a descent direction of $\hat{g}_{j}(x) ; j \in \Lambda^{\text {ac }}(x)$ at $x \in S$.

Since $X$ is an open subset of $\mathbb{R}^{n}$ and $x \in X$, there exists a $\delta_{0}>0$ such that $x+\alpha d \in X$ for $\alpha \in\left(0, \delta_{0}\right)$. Therefore from Definition 3.2, $\exists \delta_{1}>0$ such that $\hat{g}_{j}(x+\alpha d) \prec \hat{g}_{j}(x) \preceq \hat{0} \forall j \in \Lambda^{\mathrm{ac}}(x), \forall \alpha \in\left(0, \delta_{1}\right)$.

Further $\hat{g}_{i}(x) \prec \hat{0} \forall i \in \Lambda^{\text {in }}(x)$. Since $\hat{g}_{i}(x) ; i \in \Lambda^{\text {in }}(x)$ are continuous at $x, \exists \delta_{2}>0$ such that $\hat{g}_{i}(x+\alpha d) \prec$ $\hat{0} \forall i \in \Lambda^{\text {in }}(x), \forall \alpha \in\left(0, \delta_{2}\right)$.

Choose $\delta_{3}:=\min \left\{\delta_{0}, \delta_{1}, \delta_{2}\right\}$. Thus $x+\alpha d \in S \forall \alpha \in\left(0, \delta_{3}\right)$. Hence $d \in \mathscr{F}(x)$. Thus the result follows.

Corollary 4.2. If $x^{*}$ is a local weak efficient solution of $(\widehat{\mathrm{CP}})_{1}$, then $\overline{\mathscr{F}}\left(x^{*}\right) \bigcap \mathscr{D}_{1}\left(x^{*}\right)=\phi$.

Proof. From Theorem 3.8, $\mathscr{F}\left(x^{*}\right) \bigcap \mathscr{D}_{1}\left(x^{*}\right)=\phi$ at the local weak efficient solution $x^{*}$. From Lemma 4.1, $\overline{\mathscr{F}}\left(x^{*}\right) \subseteq \mathscr{F}\left(x^{*}\right)$. Therefore $\overline{\mathscr{F}}\left(x^{*}\right) \bigcap \mathscr{D}_{1}\left(x^{*}\right)=\phi$.

Theorem 4.3. If $x^{*} \in S$ is a local weak efficient solution of $(\widehat{\mathrm{CP}})_{1}$, then $\left\{d \in \mathbb{R}^{n}: d^{T} \nabla \hat{f}\left(x^{*}\right) \prec \hat{0}\right.$; $\left.d^{T} \nabla \hat{g}_{j}\left(x^{*}\right) \prec \hat{0} ; j \in \Lambda^{a c}\left(x^{*}\right)\right\}=\phi$.

Proof. Suppose there exists $d \in \mathbb{R}^{n}$ such that $d^{T} \nabla \hat{f}\left(x^{*}\right) \prec \hat{0} ; d^{T} \nabla \hat{g}_{j}\left(x^{*}\right) \prec \hat{0} ; j \in \Lambda^{\text {ac }}\left(x^{*}\right)$. Theorem 3.4 holds for $\hat{f}, \hat{g}_{j} ; j \in \Lambda^{\text {ac }}\left(x^{*}\right)$ at $x^{*} \in S$. Hence $d$ is a descent direction of $\hat{f}, \hat{g}_{j} ; j \in \Lambda^{\text {ac }}\left(x^{*}\right)$ at $x^{*}$. Since $X$ is an open subset of $\mathbb{R}^{n}$ and $x \in X$, there exists a $\delta_{0}>0$ such that $x+\alpha d \in X$ for $\alpha \in\left(0, \delta_{0}\right)$.

Therefore $\exists \delta_{1}, \delta_{2}>0$ such that $\hat{f}\left(x^{*}+\alpha d\right) \prec \hat{f}\left(x^{*}\right) \forall \alpha \in\left(0, \delta_{1}\right)$ and $\hat{g}_{j}\left(x^{*}+\alpha d\right) \prec \hat{g}_{j}\left(x^{*}\right) \prec \hat{0} \forall j \in \Lambda^{\text {ac }}\left(x^{*}\right)$, $\forall \alpha \in\left(0, \delta_{2}\right)$.

Further $\hat{g}_{j}\left(x^{*}\right) \prec \hat{0} \forall j \in \Lambda^{\text {in }}\left(x^{*}\right)$. Since $\hat{g}_{j}\left(x^{*}\right) ; j \in \Lambda^{\text {in }}\left(x^{*}\right)$ are continuous at $x^{*}, \exists \delta_{3}>0$ such that $\hat{g}_{j}\left(x^{*}+\alpha d\right) \prec \hat{0} \forall j \in \Lambda^{\text {in }}\left(x^{*}\right), \forall \alpha \in\left(0, \delta_{3}\right)$.

Choose $\delta:=\min \left\{\delta_{0}, \delta_{1}, \delta_{2}, \delta_{3}\right\}$. Therefore $\forall \alpha \in(0, \delta), \exists x+\alpha d \in S$ such that $\hat{f}\left(x^{*}+\alpha d\right) \prec \hat{f}\left(x^{*}\right)$ holds which contradicts that $x^{*} \in S$ is a local weak efficient solution of $(\widehat{\mathrm{CP}})_{1}$. Hence the result follows.

\subsubsection{Fritz-John point}

Theorem 4.4. If $x^{*}$ is a local weak efficient solution of $(\widehat{\mathrm{CP}})_{1}$, then there exist $\alpha, \beta, \gamma_{j}, \delta_{j} \geq 0, j \in \Lambda^{a c}\left(x^{*}\right)$ with $\left(\alpha, \beta, \gamma_{j}, \delta_{j}\right) \neq(0,0,0,0)$ such that the following conditions hold.

$$
\begin{aligned}
\alpha p+\beta q+\sum_{j \in \Lambda^{a c}\left(x^{*}\right)} \gamma_{j} r_{j}+\sum_{j \in \Lambda^{a c}\left(x^{*}\right)} \delta_{j} s_{j} & =0 \\
\gamma_{j} \underline{g}_{j}\left(x^{*}\right)+\delta_{j} \bar{g}_{j}\left(x^{*}\right) & =0, \quad j \in \Lambda^{a c}\left(x^{*}\right)
\end{aligned}
$$

where $p_{i}, q_{i} \in\left\{\left(\frac{\partial \hat{f}\left(x^{*}\right)}{\partial x_{i}}\right)_{\min },\left(\frac{\partial \hat{f}\left(x^{*}\right)}{\partial x_{i}}\right)_{\max }\right\}$ with $p_{i} \neq q_{i}$ and $r_{i j}, s_{i j} \in\left\{\left(\frac{\partial \hat{g_{j}}\left(x^{*}\right)}{\partial x_{i}}\right)_{\min },\left(\frac{\partial \hat{g}_{j}\left(x^{*}\right)}{\partial x_{i}}\right)_{\max }\right\}$, $j \in \Lambda^{a c}\left(x^{*}\right)$ with $r_{i j} \neq s_{i j}$. 
Proof. From Theorem 4.3, the system $\nabla \hat{f}\left(x^{*}\right)^{T} d \prec \hat{0}, \nabla \hat{g}_{j}\left(x^{*}\right)^{T} d \prec \hat{0}, j \in \Lambda^{\text {ac }}\left(x^{*}\right)$ has no solution. That is, $\sum_{i} d_{i} \frac{\partial \hat{f}\left(x^{*}\right)}{\partial x_{i}} \prec \hat{0}, \sum_{i} d_{i} \frac{\partial \hat{g}_{j}\left(x^{*}\right)}{\partial x_{i}} \prec \hat{0}, j \in \Lambda^{\text {ac }}\left(x^{*}\right)$ has no solution. This implies for $j \in \Lambda^{\text {ac }}\left(x^{*}\right)$, following system has no solution.

$$
\begin{gathered}
\sum_{i} \min \left\{d_{i}\left(\frac{\partial \hat{f}\left(x^{*}\right)}{\partial x_{i}}\right)_{\min }, d_{i}\left(\frac{\partial \hat{f}\left(x^{*}\right)}{\partial x_{i}}\right)_{\max }\right\}<0, \quad \sum_{i} \max \left\{d_{i}\left(\frac{\partial \hat{f}\left(x^{*}\right)}{\partial x_{i}}\right)_{\min }, d_{i}\left(\frac{\partial \hat{f}\left(x^{*}\right)}{\partial x_{i}}\right)_{\max }\right\}<0 \\
\sum_{i} \min \left\{d_{i}\left(\frac{\partial \hat{g}_{j}\left(x^{*}\right)}{\partial x_{i}}\right)_{\min }, d_{i}\left(\frac{\partial \hat{g}_{j}\left(x^{*}\right)}{\partial x_{i}}\right)_{\max }\right\}<0, \quad \sum_{i} \max \left\{d_{i}\left(\frac{\partial \hat{g}_{j}\left(x^{*}\right)}{\partial x_{i}}\right)_{\min }, d_{i}\left(\frac{\partial \hat{g}_{j}\left(x^{*}\right)}{\partial x_{i}}\right)_{\max }\right\}<0 .
\end{gathered}
$$

Therefore the set $\left\{\sum_{i} d_{i} p_{i}<0, \sum_{i} d_{i} q_{i}<0 ; \sum_{i} d_{i} r_{i j}<0, \sum_{i} d_{i} s_{i j}<0, j \in \Lambda^{\text {ac }}\left(x^{*}\right)\right\}$ has no solution, where $p_{i}, q_{i} \in\left\{\left(\frac{\partial \hat{f}\left(x^{*}\right)}{\partial x_{i}}\right)_{\text {min }},\left(\frac{\partial \hat{f}\left(x^{*}\right)}{\partial x_{i}}\right)_{\text {max }}\right\}$ with $p_{i} \neq q_{i}$ and $r_{i j}, s_{i j} \in\left\{\left(\frac{\partial \hat{g}_{j}\left(x^{*}\right)}{\partial x_{i}}\right)_{\text {min }},\left(\frac{\partial \hat{g_{j}}\left(x^{*}\right)}{\partial x_{i}}\right)_{\text {max }}\right\}, j \in \Lambda^{\mathrm{ac}}\left(x^{*}\right)$ with $r_{i j} \neq s_{i j}$.

This can be expressed in matrix form as $\left\{d \in \mathbb{R}^{n}: \mathscr{A}\left(x^{*}\right) d<0\right\}=\phi$, where the matrix

$$
\mathscr{A}\left(x^{*}\right)=\left(\begin{array}{cc}
p^{T} & \\
q^{T} & \\
r_{j}^{T}, & j \in \Lambda^{\mathrm{ac}}\left(x^{*}\right) \\
s_{j}^{T}, & { }^{\prime \prime}
\end{array}\right)_{\left(2+2\left|\Lambda^{\mathrm{ac}}\left(x^{*}\right)\right|\right) \times n} .
$$

Using Gordan's theorem of alternative, there exist $\alpha, \beta, \gamma_{j}, \delta_{j} \geq 0, j \in \Lambda^{\mathrm{ac}}\left(x^{*}\right)$ with $\left(\alpha, \beta, \gamma_{j}, \delta_{j}\right) \neq(0,0,0,0)$ such that

$$
\alpha p+\beta q+\sum_{j \in \Lambda^{\mathrm{ac}}\left(x^{*}\right)} \gamma_{j} r_{j}+\sum_{j \in \Lambda^{\mathrm{ac}}\left(x^{*}\right)} \delta_{j} s_{j}=0 .
$$

Since $x^{*} \in S, \hat{g}_{j}\left(x^{*}\right) \preceq \hat{0}$ and for $j \in \Lambda^{\text {ac }}\left(x^{*}\right), 0 \in \hat{g}_{j}\left(x^{*}\right)$. Therefore either (i) $\underline{g}_{j}\left(x^{*}\right)<0, \bar{g}_{j}\left(x^{*}\right)=0$ or (ii) $\underline{g}_{j}\left(x^{*}\right)=0=\bar{g}_{j}\left(x^{*}\right)$. (4.2) holds for $\gamma_{j}=0 ; \delta_{j} \geq 0$ in case (i) and $\gamma_{j} \geq 0 ; \delta_{j} \geq 0$ in case (ii).

The necessary conditions (4.1) and (4.2) in Theorem 4.4 become the Fritz-John necessary conditions of a general optimization problem if the parameters of $(\widehat{\mathrm{CP}})_{1}$ are degenerate intervals. Hence we say the point $x^{*}$ satisfying (4.1) and (4.2) as Fritz-John point.

Definition 4.5 (Fritz-John point). A point $x^{*} \in S$ is said to be Fritz-John point of $(\widehat{\mathrm{CP}})_{1}$ if there exist $\alpha, \beta, \gamma_{j}, \delta_{j} \geq 0, j \in \Lambda^{\mathrm{ac}}\left(x^{*}\right)$ with $\left(\alpha, \beta, \gamma_{j}, \delta_{j}\right) \neq(0,0,0,0)$ such that the conditions (4.1) and (4.2) hold.

Example 4.6. $x^{*}=(2,1)^{T}$ as a Fritz-John point of the following interval valued inequality constrained minimization problem.

$$
\begin{aligned}
\text { Min } \quad & {[1,3]\left(x_{1}-3\right)^{2} \oplus[2,4]\left(x_{2}-2\right)^{2} } \\
\text { Subject to } \quad & {\left[\frac{1}{2}, 1\right] x_{1}^{2} \oplus\left[\frac{1}{3}, 1\right] x_{2}^{2} \preceq[3,5] } \\
& {[1,2] x_{1} \oplus[2,3] x_{2} \preceq[4,7] } \\
& x_{1} \geq 0, x_{2} \geq 0 .
\end{aligned}
$$

Here $\hat{g}_{1}(x):=\left[\frac{1}{2}, 1\right] x_{1}^{2} \oplus\left[\frac{1}{3}, 1\right] x_{2}^{2} \ominus_{\mathrm{gH}}[3,5] ; \hat{g}_{2}(x):=[1,2] x_{1} \oplus[2,3] x_{2} \ominus_{\mathrm{gH}}[4,7] ; \hat{g}_{3}(x):=[-1,-1] x_{1}$ and $\hat{g}_{4}(x):=[-1,-1] x_{2}$. Following calculations justify that $x^{*}=(2,1)^{T}$ is a Fritz-John point of this problem. 
Note that $0 \in \hat{g}_{1}\left(x^{*}\right)$ with $\underline{g}_{1}\left(x^{*}\right)<0, \bar{g}_{1}\left(x^{*}\right)=0 ; \hat{g}_{2}\left(x^{*}\right)=\hat{0} ; \hat{g}_{3}\left(x^{*}\right) \prec \hat{0}$ and $\hat{g}_{4}\left(x^{*}\right) \prec \hat{0}$. Therefore $\Lambda^{\text {ac }}\left(x^{*}\right)=\{1,2\}$. Here, $\hat{f}, \hat{g}_{1}, \hat{g}_{2}$ are gH differentiable at $x^{*}$.

$$
\begin{aligned}
\frac{\partial \hat{f}\left(x^{*}\right)}{\partial x_{1}} & =[-6,-2], \quad \frac{\partial \hat{f}\left(x^{*}\right)}{\partial x_{2}}=[-8,-4], \quad \frac{\partial \hat{g}_{1}\left(x^{*}\right)}{\partial x_{1}}=[2,4] \\
\frac{\partial \hat{g}_{1}\left(x^{*}\right)}{\partial x_{2}} & =\left[\frac{2}{3}, 2\right] x_{2}^{*}=\left[\frac{2}{3}, 2\right], \quad \frac{\partial \hat{g}_{2}\left(x^{*}\right)}{\partial x_{1}}=[1,2], \quad \frac{\partial \hat{g}_{2}\left(x^{*}\right)}{\partial x_{2}}=[2,3] .
\end{aligned}
$$

For $d \in \mathbb{R}^{n}$, consider the system of interval inequalities $\nabla \hat{f}\left(x^{*}\right)^{T} d \prec \hat{0} ; \nabla \hat{g}_{j}\left(x^{*}\right)^{T} d \prec \hat{0}, j \in \Lambda^{\text {ac }}\left(x^{*}\right)$, which is

$$
[-6,-2] d_{1} \oplus[-8-4] d_{2} \prec \hat{0}, \quad[2,4] d_{1} \oplus\left[\frac{2}{3}, 2\right] d_{2} \prec \hat{0}, \quad[1,2] d_{1} \oplus[2,3] d_{2} \prec \hat{0} .
$$

Case 1. If $d_{1}, d_{2}$ are of same sign then (4.3) becomes

$$
-6 d_{1}-8 d_{2}<0 ; \quad-2 d_{1}-4 d_{2}<0 ; \quad 2 d_{1}+\frac{2}{3} d_{2}<0 ; \quad 4 d_{1}+2 d_{2}<0 ; \quad d_{1}+2 d_{2}<0 ; \quad 2 d_{1}+3 d_{2}<0,
$$

which has no solution. By theorem of alternative, the following system has a solution $\left(\alpha=1, \beta=1, \gamma_{1}=0\right.$, $\gamma_{2}=\frac{16}{3}, \delta_{1}=\frac{2}{3}, \delta_{2}=0$ is the solution).

$$
\begin{aligned}
\alpha\left(\begin{array}{l}
-6 \\
-8
\end{array}\right)+\beta\left(\begin{array}{l}
-2 \\
-4
\end{array}\right)+\gamma_{1}\left(\begin{array}{l}
2 \\
\frac{2}{3}
\end{array}\right)+\delta_{1}\left(\begin{array}{l}
4 \\
2
\end{array}\right)+\gamma_{2}\left(\begin{array}{l}
1 \\
2
\end{array}\right)+\delta_{2}\left(\begin{array}{l}
2 \\
3
\end{array}\right) & =0 \\
-\frac{2}{3} \gamma_{1}+0 \delta_{1} & =0 \\
0 \gamma_{2}+0 \delta_{2} & =0 .
\end{aligned}
$$

Case 2. If $d_{1}, d_{2}$ are of opposite sign then (4.3) becomes

$$
-6 d_{1}-4 d_{2}<0 ; \quad-2 d_{1}-8 d_{2}<0 ; \quad 2 d_{1}+2 d_{2}<0 ; \quad 4 d_{1}+\frac{2}{3} d_{2}<0 ; \quad d_{1}+3 d_{2}<0 ; \quad 2 d_{1}+2 d_{2}<0,
$$

which has no solution. By theorem of alternative, the following system has a solution $\left(\alpha=1, \beta=1, \gamma_{1}=0\right.$, $\gamma_{2}=\frac{64}{17}, \delta_{1}=\frac{18}{17}, \delta_{2}=0$ is the solution).

$$
\begin{aligned}
\alpha\left(\begin{array}{l}
-6 \\
-4
\end{array}\right)+\beta\left(\begin{array}{l}
-2 \\
-8
\end{array}\right)+\gamma_{1}\left(\begin{array}{l}
2 \\
2
\end{array}\right)+\delta_{1}\left(\begin{array}{l}
4 \\
\frac{2}{3}
\end{array}\right)+\gamma_{2}\left(\begin{array}{l}
1 \\
3
\end{array}\right)+\delta_{2}\left(\begin{array}{l}
2 \\
2
\end{array}\right) & =0 \\
-\frac{2}{3} \gamma_{1}+0 \delta_{1} & =0 \\
0 \gamma_{2}+0 \delta_{2} & =0 .
\end{aligned}
$$

Hence the conditions (4.1) and (4.2) are satisfied and the feasible point $x^{*}=(2,1)^{T}$ is Fritz-John point.

\subsubsection{Regularity conditions and Karush-Kuhn-Tucker point}

In a classical optimization model, Karush-Kuhn-Tucker (KKT hereafter) conditions, associated with non zero multiplier of the objective function, are obtained by imposing some constraints qualification. As in general non linear programming it is necessary to explore some regularity conditions in interval sense as constraint qualifications, which is studied in this article using the linear independence of interval vectors.

Definition 4.7 (Linear independence of interval vectors, [3, 4]). The set of $n$ different interval vectors $\left\{\hat{u}^{(1)}, \hat{u}^{(2)}, \ldots, \hat{u}^{(n)}: \hat{u}^{(i)} \neq \hat{u}^{(j)}, i, j \in \Lambda_{n}\right\}$ is called linearly independent if the set of real vectors $\left\{u^{(1)}, u^{(2)}, \ldots, u^{(n)}: u^{(i)} \in \hat{u}^{(i)} \forall i \in \Lambda_{n}\right\}$ is linearly independent.

Otherwise if there exists at least one set of linearly dependent real vectors $\left\{v^{(1)}, v^{(2)}, \ldots, v^{(n)}: v^{(i)} \in \hat{u}^{(i)} \forall i \in \Lambda_{n}\right\}$, then the set of interval vectors $\left\{\hat{u}^{(1)}, \hat{u}^{(2)}, \ldots, \hat{u}^{(n)}\right\}$ is linearly dependent. 
Note 4.8. The following results from [3] are summarized which are useful to determine the linear dependence or independence of interval vectors.

(1) Given $n+1$ interval vectors, assume that the set of $\mathrm{n}$ interval vectors $\left\{\hat{u}^{(1)}, \hat{u}^{(2)}, \ldots, \hat{u}^{(n)}\right\}$ is linearly independent. Then the set of interval vectors $\left\{\hat{u}^{(i)}, i=1,2, \ldots, n+1\right\}$ is linearly dependent if and only if there exists a real vector comprised of $b_{i}, i=1,2, \ldots, n$ that satisfies $0_{n} \in \hat{u}^{(n+1)} \oplus(-1)\left(\sum_{i=1}^{n} b_{i} \hat{u}^{(i)}\right)$ for at least one particular sign of $b_{i}$.

(2) Based on the assumption of above result 1, if $\hat{u}_{n+1}$ is linearly dependent on the other interval vectors $\hat{u}_{i}, i=1,1, \ldots, n$, then $\left(\hat{u}_{n+1} \cap \sum_{i=1}^{n} b_{i} \hat{u}^{(i)}\right) \neq \phi$

(3) Given $n+1$ interval vectors, assume that the set of $n$ interval vectors $\left\{\hat{u}^{(1)}, \hat{u}^{(2)}, \ldots, \hat{u}^{(n)}\right\}$ is linearly independent. If $0_{n} \in \hat{u}^{(n+1)}$, then $\hat{u}^{(n+1)}$ is linearly dependent on that set of interval vectors.

(4) From the result 3, one may conclude that $\left\{\hat{u}^{(n)}\right\}$ is linearly independent if $0_{n} \notin \hat{u}^{(n)}$.

In the light of the concept of constraint qualifications for a general constrained nonlinear optimization problem, one can say that $(\widehat{\mathrm{CP}})_{1}$ satisfies the constraint qualification at $x^{*}$ if the set $\left\{\nabla \hat{g}_{j}\left(x^{*}\right): j \in \Lambda^{\text {ac }}\left(x^{*}\right)\right\}$ is linearly independent. We call the point satisfying this condition as a regular point.

Theorem 4.9. Suppose $x^{*} \in S$ is a local weak efficient solution of $(\widehat{\mathrm{CP}})_{1}$ and the set $\left\{\nabla \hat{g}_{j}\left(x^{*}\right): j \in \Lambda^{a c}\left(x^{*}\right)\right\}$ is linearly independent. Then at least one of $\alpha, \beta$ in (4.1) will be nonzero.

Proof. Suppose $\alpha=0=\beta$ in (4.1). Then

$$
\sum_{j \in \Lambda^{\mathrm{ac}}\left(x^{*}\right)}\left(\gamma_{j} r_{j}+\delta_{j} s_{j}\right)=0
$$

where $r_{i j}, s_{i j} \in\left\{\left(\frac{\partial \hat{g}_{j}\left(x^{*}\right)}{\partial x_{i}}\right)_{\min },\left(\frac{\partial \hat{g}_{j}\left(x^{*}\right)}{\partial x_{i}}\right)_{\max }\right\}, j \in \Lambda^{\text {ac }}\left(x^{*}\right)$ with $r_{i j} \neq s_{i j}$.

Since $\alpha=0=\beta$, from Theorem 4.4, $\left(\gamma_{j}, \delta_{j}\right) \neq(0,0)$ for at least one $j \in \Lambda^{\text {ac }}\left(x^{*}\right)$. Hence $\gamma_{j}+\delta_{j}>0$ for at least one $j \in \Lambda^{\text {ac }}\left(x^{*}\right)$. From (4.4),

$$
\sum_{j \in \Lambda^{\mathrm{ac}}\left(x^{*}\right)}\left(\gamma_{j}+\delta_{j}\right)\left(\frac{\gamma_{j} r_{j}+\delta_{j} s_{j}}{\gamma_{j}+\delta_{j}}\right)=0 \quad \text { since } \quad\left(\gamma_{j}+\delta_{j}\right)>0 .
$$

$0 \leq \frac{\gamma_{j}}{\gamma_{j}+\delta_{j}}, \frac{\delta_{j}}{\gamma_{j}+\delta_{j}} \leq 1$ and $\frac{\gamma_{j}}{\gamma_{j}+\delta_{j}}+\frac{\delta_{j}}{\gamma_{j}+\delta_{j}}=1$. Therefore for each $i \in \Lambda_{n}$,

$$
\left(\frac{\partial \hat{g}_{j}\left(x^{*}\right)}{\partial x_{i}}\right)_{\min } \leq\left(\frac{\gamma_{j} r_{j}+\delta_{j} s_{j}}{\gamma_{j}+\delta_{j}}\right) \leq\left(\frac{\partial \hat{g}_{j}\left(x^{*}\right)}{\partial x_{i}}\right)_{\max }, \quad j \in \Lambda^{\mathrm{ac}}\left(x^{*}\right) .
$$

Hence $\forall i$, if we consider $v_{j}:=\left(\frac{\gamma_{j} r_{j}+\delta_{j} s_{j}}{\gamma_{j}+\delta_{j}}\right) ; j \in \Lambda^{\text {ac }}\left(x^{*}\right)$, then $v_{j} \in \nabla \hat{g}_{j}\left(x^{*}\right)$ for each $j \in \Lambda^{\text {ac }}\left(x^{*}\right)$.

Therefore from (4.5), $\sum_{j \in \Lambda^{\mathrm{ac}}\left(x^{*}\right)}\left(\gamma_{j}+\delta_{j}\right) v_{j}=0 ; v_{j} \in \nabla \hat{g}_{j}\left(x^{*}\right)$ with $\left(\gamma_{j}+\delta_{j}\right)>0$ implies the set of interval vectors $\left\{\nabla \hat{g}_{j}\left(x^{*}\right): j \in \Lambda^{\text {ac }}\left(x^{*}\right)\right\}$ is linearly dependent which is a contradiction. Therefore $\alpha, \beta \geq 0$ with $(\alpha, \beta) \neq(0,0)$.

From Theorem 4.4, one may note that if $x^{*} \in S$ is a local weak efficient solution of $(\widehat{\mathrm{CP}})_{1}$, then there exist $\alpha, \beta, \gamma_{j}, \delta_{j} \geq 0, j \in \Lambda^{\mathrm{ac}}\left(x^{*}\right)$ with $\left(\alpha, \beta, \gamma_{j}, \delta_{j}\right) \neq(0,0,0,0)$ such that the conditions (4.1) and (4.2) hold. Under the same assumption, from Theorem 4.9 one may note that if the set $\left\{\nabla \hat{g}_{j}\left(x^{*}\right): j \in \Lambda^{\text {ac }}\left(x^{*}\right)\right\}$ is linearly independent, then at least one of $\alpha, \beta$ in (4.1) will be nonzero. Therefore we say the conditions (4.1) and (4.2) satisfying $(\alpha, \beta) \neq(0,0)$ as Karush-Kuhn-Tucker necessary optimality conditions for $(\widehat{\mathrm{CP}})_{1}$ and the point $x^{*}$ as a KKT point of $(\widehat{\mathrm{CP}})_{1}$. 
Definition 4.10 (Karush-Kuhn-Tucker point). A point $x^{*} \in S$ is said to be Karush-Kuhn-Tucker point of $(\widehat{\mathrm{CP}})_{1}$ if there exist $\alpha, \beta, \gamma_{j}, \delta_{j} \geq 0, j \in \Lambda^{\mathrm{ac}}\left(x^{*}\right)$ with $(\alpha, \beta) \neq(0,0)$ such that the conditions (4.1) and (4.2) hold.

Example 4.11 (Verification for KKT point). $x^{*}=(0.5,0)^{T}$ is the KKT point of the following problem.

$$
\begin{aligned}
\text { Min } & {[-10,-6] x_{1} \oplus[2,3] x_{2} \oplus[4,10] x_{1}^{2} \oplus[-1,-1] x_{1} x_{2} \oplus[10,20] x_{2}^{2} } \\
\text { Subject to } & {[1,2] x_{1} \oplus[3,3] x_{2} \preceq[1,10] } \\
& {[-2,8] x_{1} \oplus[4,6] x_{2} \preceq[4,16] } \\
& x_{1} \geq 0, x_{2} \geq 0 .
\end{aligned}
$$

Here $\hat{g}_{1}(x):=[1,2] x_{1} \oplus[3,3] x_{2} \ominus_{\mathrm{gH}}[1,10] ; \hat{g}_{2}(x):=[-2,8] x_{1} \oplus[4,6] x_{2} \ominus_{\mathrm{gH}}[4,6] ; \hat{g}_{3}(x):=[-1,-1] x_{1}$ and $\hat{g}_{4}(x):=[-1,-1] x_{2}$. Here $\hat{g}_{1}\left(x^{*}\right) \prec \hat{0} ; \hat{g}_{2}\left(x^{*}\right) \prec \hat{0} ; \hat{g}_{3}\left(x^{*}\right) \prec \hat{0}$ and $\hat{g}_{4}\left(x^{*}\right)=\hat{0}$.

Therefore $\Lambda^{\text {ac }}\left(x^{*}\right)=\{4\}$. It is easy to verify that $\hat{f}, \hat{g}_{4}$ are gH differentiable at $x^{*} \cdot \frac{\partial \hat{f}\left(x^{*}\right)}{\partial x_{1}}=[-6,4] ; \frac{\partial \hat{f}\left(x^{*}\right)}{\partial x_{2}}=$ $[1.5,3.5]$ and $\frac{\partial \hat{g}_{4}\left(x^{*}\right)}{\partial x_{1}}=\hat{0} ; \frac{\partial \hat{g}_{4}\left(x^{*}\right)}{\partial x_{2}}=[-1,-1]$.

For $d \in \mathbb{R}^{n}$, consider the system of interval inequalities $\nabla \hat{f}\left(x^{*}\right)^{T} d \prec \hat{0} ; \nabla \hat{g}_{j}\left(x^{*}\right)^{T} d \prec \hat{0}, j \in \Lambda^{\text {ac }}\left(x^{*}\right)$, which is same as

$$
[-6,4] d_{1} \oplus[1.5,3.5] d_{2} \prec \hat{0}, \quad[0,0] d_{1} \oplus[-1,-1] d_{2} \prec \hat{0} .
$$

If $d_{1}, d_{2}$ are of same sign, then the following system has no solution.

$$
-6 d_{1}+1.5 d_{2}<0 ; \quad 4 d_{1}+3.5 d_{2}<0 ; \quad 0 . d_{1}-d_{2}<0 .
$$

Taking $\alpha=2, \beta=3, \gamma_{4}=13.5$ we see that the following system has solution.

$$
\begin{aligned}
\alpha\left(\begin{array}{c}
-6 \\
1.5
\end{array}\right)+\beta\left(\begin{array}{c}
4 \\
3.5
\end{array}\right)+\gamma_{4}\left(\begin{array}{l}
0 \\
1
\end{array}\right) & =0 \\
0 \gamma_{4} & =0 .
\end{aligned}
$$

If $d_{1}, d_{2}$ are of opposite sign, then the following system has no solution.

$$
-6 d_{1}+3.5 d_{2}<0 ; \quad 4 d_{1}+1.5 d_{2}<0 ; \quad 0 d_{1}-d_{2}<0 .
$$

Taking $\alpha=2, \beta=3, \gamma_{1}=11.5$ we see that the following system has solution.

$$
\begin{aligned}
\alpha\left(\begin{array}{c}
-6 \\
3.5
\end{array}\right)+\beta\left(\begin{array}{c}
4 \\
1.5
\end{array}\right)+\gamma_{4}\left(\begin{array}{l}
0 \\
1
\end{array}\right) & =0 \\
0 \gamma_{4} & =0 .
\end{aligned}
$$

Here $\nabla \hat{g}_{4}(0.5,0)=(\hat{0},[-1,-1])^{T}$. Clearly $(0,0)^{T} \notin \nabla \hat{g}_{4}(0.5,0)$. The singleton set of interval vector not containing zero vector is linearly independent. Hence $x^{*}=(0.5,0)^{T}$ is KKT point.

The following example shows that Fritz-John point need not be KKT point for $(\widehat{\mathrm{CP}})_{1}$.

Example 4.12. $x^{*}=(2,1)^{T}$ is a Fritz-John point but not be a KKT point of Example 4.6.

Consider the set $\left\{\nabla \hat{g}_{1}\left(x^{*}\right), \nabla \hat{g}_{2}\left(x^{*}\right)\right\}$ to check linear independence. For $b_{1}=1,0 \in \frac{\partial \hat{g}_{1}\left(x^{*}\right)}{\partial x_{1}} \oplus(-1) \frac{\partial \hat{g}_{2}\left(x^{*}\right)}{\partial x_{1}}=$ $[2,4] \oplus(-1)[1,2]=[0,3]$ and $0 \in \frac{\partial \hat{g}_{1}\left(x^{*}\right)}{\partial x_{2}} \oplus(-1) \frac{\partial \hat{g}_{2}\left(x^{*}\right)}{\partial x_{2}}=\left[\frac{2}{3}, 2\right] \oplus(-1)[2,3]=\left[-\frac{7}{3}, 0\right]$. Also one may note that $\nabla \hat{g}_{1}\left(x^{*}\right)$ as well as $\nabla \hat{g}_{2}\left(x^{*}\right)$ contain $(2,2)^{T}$. Hence using Result (1) and (2) of Note 4.8 , the set is linearly dependent. $x^{*}=(2,1)^{T}$ is not a KKT point of the problem. 


\subsection{Sufficient condition using gH-pseudo convexity}

Theorem 4.13. Suppose $\hat{f}$ is $\mathrm{gH}-$ pseudo convex at $x^{*} \in S$ and the following condition holds for each $j \in \Lambda^{a c}\left(x^{*}\right)$,

$$
\left(x-x^{*}\right)^{T} \nabla \hat{g}_{j}\left(x^{*}\right) \underset{0}{=} \quad \forall x \in S .
$$

If $x^{*}$ is a Karush-Kuhn-Tucker point of $(\widehat{\mathrm{CP}})_{1}$, then $x^{*}$ is a local weak efficient solution for $(\widehat{\mathrm{CP}})_{1}$.

Proof. Suppose $x^{*} \in S$ be not a weak efficient solution of $(\widehat{\mathrm{CP}})_{1}$. Then there exists another $y^{*} \in S$ such that $\hat{f}\left(y^{*}\right) \prec \hat{f}\left(x^{*}\right)$ holds. Since $\hat{f}$ is gH-pseudo convex at $x^{*} \in S$ and condition (4.6) hold for each $j \in \Lambda^{\text {ac }}\left(x^{*}\right)$,

$$
\left(y^{*}-x^{*}\right)^{T} \nabla \hat{f}\left(x^{*}\right) \prec \hat{0} ; \quad\left(y^{*}-x^{*}\right)^{T} \nabla \hat{g}_{j}\left(x^{*}\right) \underset{\hat{0}}{=}, \quad j \in \Lambda^{\mathrm{ac}}\left(x^{*}\right)
$$

Let $d^{*}=y^{*}-x^{*}$. Then the following systems have solution for $d \in \mathbb{R}^{n}$.

$$
\sum_{i} d_{i} p_{i}<0, \quad \sum_{i} d_{i} q_{i}<0 ; \quad \sum_{i} d_{i} r_{i j} \leq 0, \quad \sum_{i} d_{i} s_{i j} \leq 0 ; \quad j \in \Lambda^{\mathrm{ac}}\left(x^{*}\right)
$$

where $p_{i}, q_{i} \in\left\{\left(\frac{\partial \hat{f}\left(x^{*}\right)}{\partial x_{i}}\right)_{\min },\left(\frac{\partial \hat{f}\left(x^{*}\right)}{\partial x_{i}}\right)_{\max }\right\}$ with $p_{i} \neq q_{i}$ and $r_{i j}, s_{i j} \in\left\{\left(\frac{\partial \hat{g}_{j}\left(x^{*}\right)}{\partial x_{i}}\right)_{\min },\left(\frac{\partial \hat{g}_{j}\left(x^{*}\right)}{\partial x_{i}}\right)_{\max }\right\}$, $j \in \Lambda^{\text {ac }}\left(x^{*}\right)$ with $r_{i j} \neq s_{i j}$.

Then using Gordan's theorem of alternative there exist no $\alpha, \beta, \gamma_{j}, \delta_{j} \geq 0 ; j \in \Lambda^{\text {ac }}\left(x^{*}\right)$ such that (4.1) holds. Therefore $x^{*}$ cannot be a Karush-Kuhn-Tucker point of $(\widehat{\mathrm{CP}})_{1}$ which contradicts our hypothesis. Hence $x^{*} \in S$ is a weak efficient solution of $(\widehat{\mathrm{CP}})_{1}$.

Example 4.14. $x^{*}=(0,0)^{T}$ is a weak efficient solution of the following problem.

$$
\begin{aligned}
\text { Min } & {[1,4] x_{1}^{2} \oplus[2,4] x_{2} } \\
\text { Subject to } & {[1,3] x_{2}^{3} \oplus[-2,-1] x_{2} \oplus[1,1] x_{1} \supseteqq \hat{0} } \\
& x_{2} \geq 0 .
\end{aligned}
$$

Here $\hat{g}_{1}(x):=[1,3] x_{2}^{3} \oplus[-2,-1] x_{2} \oplus[1,1] x_{1}$ and $\hat{g}_{2}(x):=[-1,-1] x_{2}$. At $x^{*}, \hat{g}_{1}\left(x^{*}\right)=\hat{0}=\hat{g}_{2}\left(x^{*}\right)$. Therefore $\Lambda^{\text {ac }}=\{1,2\} . \hat{f}, \hat{g}_{1}, \hat{g}_{2}$ all are gH-differentiable at $x^{*}=(0,0)^{T} . \nabla \hat{g}_{1}\left(x^{*}\right)=([1,1],[-2,-1])^{T}$, $\nabla \hat{g}_{2}\left(x^{*}\right)=([0,0],[-1,-1])^{T}$.

Proceeding as in Example 3.11, it can be shown that $\hat{f}$ is gH-pseudo convex at $x^{*}$. Also, $\left(y-x^{*}\right)^{T} \nabla \hat{g}_{1}\left(x^{*}\right)=$ $[1,1] y_{1} \oplus[-2,-1] y_{2} \precsim \ominus_{\mathrm{gH}}[1,3] y_{2}^{3} \prec \hat{0}$ and $\left(y-x^{*}\right)^{T} \nabla \hat{g}_{2}\left(x^{*}\right)=[-1,-1] y_{2} \precsim \hat{0}$.

Proceeding as previous Example 4.6, the following system has solution

$$
\begin{aligned}
\alpha\left(\begin{array}{l}
0 \\
2
\end{array}\right)+\beta\left(\begin{array}{l}
0 \\
4
\end{array}\right)+\gamma_{1}\left(\begin{array}{c}
1 \\
-2
\end{array}\right)+\delta_{1}\left(\begin{array}{c}
1 \\
-1
\end{array}\right)+\gamma_{2}\left(\begin{array}{c}
0 \\
-1
\end{array}\right) & =0 \\
0 \gamma_{1}+0 \delta_{1} & =0 \\
0 \gamma_{2} & =0
\end{aligned}
$$

for $\alpha=1, \beta=0, \gamma_{1}=-1, \delta_{1}=1, \gamma_{2}=3$.

The set $\left\{\nabla \hat{g}_{1}\left(x^{*}\right), \nabla \hat{g}_{2}\left(x^{*}\right)\right\}$ is a linearly independent set of two interval vectors since for $\left\{(1, a)^{T},(0,-1)^{T}\right\}$, where $-2 \leq a \leq-1$ is the linearly independent set of real vectors (see Def. 4.7).

Hence $x^{*}$ is a KKT point.

Hence by Theorem $4.13, x^{*}$ is a weak efficient solution. This can also be verified from the definition of weak efficient solution. For $x_{2} \geq 0, x_{1}^{2}+2 x_{2} \nless 0$ and $x_{1}^{2}+x_{2} \nless 0$. Hence there is no feasible point $\left(x_{1}, x_{2}\right) \in S$ such that $\hat{f}(x) \prec \hat{f}\left(x^{*}\right)=[0,0]$ hold. 


\section{Conclusions}

In this article, existence of solution for the general interval valued constrained minimization problem is studied. Later this concept is extended to derive Fritz-John and Karush-Kuhn-Tucker optimality conditions for interval valued inequality constraints. We have considered gH-differentiability of interval valued function through out this article. Hence our optimality conditions totally deal with the differentiability of interval valued function and free from the burden of differentiability of end point functions. Sufficient optimality conditions are derived using gH-pseudo convexity concept, which are not necessarily dependent on endpoint functions. Here we considered the condition of active constraints in inclusion form as $0 \in \hat{g}_{j}\left(x^{*}\right)$. The reason behind this fact is there is no point that whole interval valued constraint function will be identically zero always in case of active constraints. Since the necessary optimality conditions are totally dependent on interval valued constraint function, we have explored regularity condition for KKT necessary optimality condition using linear independence of interval vectors. Since most of the existing literatures used the real valued constraint functions or depend on upper bound functions to study regularity conditions, those can be considered as a particular instances of our present study.

Acknowledgements. The authors would like to thank the reviewers for the detailed comments and suggestions that have significantly improved the content as well as the presentation of the results in the paper.

\section{REFERENCES}

[1] I. Ahmad, D. Singh and B.A. Dar, Optimality conditions in multiobjective programming problems with interval valued objective functions. Control Cybern. 44 (2015) 19-45.

[2] I. Ahmad, D. Singh, B.A. Dar, Optimality and duality in non-differentiable interval valued multiobjective programming. Int. J. Math. Oper. Res. 11 (2017) 332-356.

[3] H.-S. Ahn, An algorithm to determine linear independence of a set of interval vectors. Appl. Math. Comput. 219 (2013) $10822-10830$.

[4] H.-S. Ahn, K.L. Moore and Y. Chen, Linear independency of interval vectors and its applications to robust controllability tests. In: Proceedings of the 44th IEEE Conference on Decision and Control. IEEE (2005) 8070-8075.

[5] A.K. Bhurjee and G. Panda, Efficient solution of interval optimization problem. Math. Methods Oper. Res. 76 (2012) $273-288$.

[6] Y. Chalco-Cano, H. Román-Flores and M.-D. Jiménez-Gamero, Generalized derivative and $\pi$-derivative for set-valued functions. Inf. Sci. 181 (2011) 2177-2188.

[7] Y. Chalco-Cano, W.A. Lodwick and A. Rufian-Lizana, Optimality conditions of type KKT for optimization problem with interval-valued objective function via generalized derivative. Fuzzy Optim. Decis. Making 12 (2013) 305-322.

[8] S. Chanas and D. Kuchta, Multiobjective programming in optimization of interval objective functions, a generalized approach. Eur. J. Oper. Res. 94 (1996) 594-598.

[9] J. Chinneck and K. Ramadan, Linear programming with interval coefficients. J. Oper. Res. Soc. 51 (2000) $209-220$.

[10] M. Hladík, Optimal value range in interval linear programming. Fuzzy Optim. Decis. Making 8 (2009) $283-294$.

[11] M. Hladík, Optimal value bounds in nonlinear programming with interval data. Top 19 (2011) 93-106.

[12] M. Hukuhara, Integration des applications mesurables dont la valeur est un compact convexe. Funkcial. Ekvac. 10 (1967) 205-223.

[13] H. Ishibuchi and H. Tanaka, Multiobjective programming in optimization of the interval objective function. Eur. J. Oper. Res. 48 (1990) 219-225.

[14] W. Li and X. Tian, Numerical solution method for general interval quadratic programming. Appl. Math. Comput. 202 (2008) $589-595$.

[15] S.-T. Liu and R.-T. Wang, A numerical smolution method to interval quadratic programming. Appl. Math. Comput. 189 (2007) 1274-1281

[16] S. Markov, Calculus for interval functions of a real variable. Computing 22 (1979) 325-337.

[17] R.E. Moore, R.B. Kearfott and M.J. Cloud, Introduction to Interval Analysis. SIAM, Philadelphia, PA (2009).

[18] C. Oliveira and C.H. Antunes, Multiple objective linear programming models with interval coefficients - an illustrated overview. Eur. J. Oper. Res. 181 (2007) 1434-1463.

[19] R. Osuna-Gómez, Y. Chalco-Cano, B. Hernández-Jiménez and G. Ruiz-Garzón, Optimality conditions for generalized differentiable interval-valued functions. Inf. Sci. 321 (2015) 136-146.

[20] R. Osuna-Gómez, B. Hernández-Jiménez, Y. Chalco-Cano and G. Ruiz-Garzón, New efficiency conditions for multiobjective interval-valued programming problems. Inf. Sci. 420 (2017) 235-248.

[21] P. Roy and G. Panda, On critical point for functions with bounded parameters. In: 2019 IEEE International Conference on Electrical, Computer and Communication Technologies (ICECCT). IEEE (2019) 1-5. 
[22] P. Roy and G. Panda, Expansion of generalized Hukuhara differentiable interval valued function. New Math. Nat. Comput. 15 (2019) 553-570.

[23] D. Singh, B.A. Dar and A. Goyal, KKT optimality conditions for interval valued optimization problems. J. Nonlinear Anal. Optim.: Theory App. 5 (2014) 91-103.

[24] D. Singh, B.A. Dar and D. Kim, KKT optimality conditions in interval valued multiobjective programming with generalized differentiable functions. Eur. J. Oper. Res. 254 (2016) 29-39.

[25] L. Stefanini, A generalization of Hukuhara difference for interval and fuzzy arithmetic. In: Vol. 48 of Soft Methods for Handling Variability and Imprecision. Series on Advances in Soft Computing. Springer, Berlin-Heidelberg (2008).

[26] L. Stefanini and B. Bede, Generalized Hukuhara differentiability of interval-valued functions and interval differential equations. Nonlinear Anal.: Theory Methods App. 71 (2009) 1311-1328.

[27] B. Urli and R. Nadeau, An interactive method to multiobjective linear programming problems with interval coefficients. INFOR: Inf. Syst. Oper. Res. 30 (1992) 127-137.

[28] H.-C. Wu, The Karush-Kuhn-Tucker optimality conditions in an optimization problem with interval-valued objective function. Eur. J. Oper. Res. 176 (2007) 46-59.

[29] H.-C. Wu, The Karush-Kuhn-Tucker optimality conditions in multiobjective programming problems with interval-valued objective functions. Eur. J. Oper. Res. 196 (2009) 49-60.

[30] J. Zhang, Q. Zheng, C. Zhou, X. Ma and L. Li, On interval-valued pseudolinear functions and interval-valued pseudolinear optimization problems. J. Funct. Spaces 2015 (2015) 610848. 